\title{
Aligning sequence from molecular inversion probes
}

\author{
Brent S. Pedersen ${ }^{1, *}$ Elissa Murphy ${ }^{1}$, \\ Ivana V. Yang ${ }^{1}$ and David A. Schwartz ${ }^{1}$ \\ ${ }^{1}$ Department of Medicine, University of Colorado Denver, School of Medicine, Denver, Colorado, \\ USA. 80045
}

Received on XXXXX; revised on XXXXX; accepted on XXXXX

\section{Associate Editor: XXXXXXX}

\section{ABSTRACT}

Summary: Molecular inversion probes (MIP's) allow efficient enrichment of genomic regions of interest for the purpose of targeted sequencing. To date, there is a paucity of simple-to-use software to align sequences derived from this method. Here, we describe a single program that performs mapping, arm removal, and deduplication before outputting alignments in SAM format.

Availability: bwa-mips is available at https://github.com/brentp/bwamips under the MIT license.

Contact: bpederse@gmail.com

\section{INTRODUCTION}

While sequencing costs have dropped dramatically, it is still prohibitive to perform entire genome sequencing on large numbers of samples. Methods such as the use of MIPs for target enrichment can reduce costs and focus efforts on genomic locations likely to yield findings of interest. In addition, the use of MIPs obviates common hurdles of standard library construction resulting in a highly scalable and cost effective method for targeted sequencing across large cohorts. MIP capture is a relatively quick and easy method involving annealing, gap-fill and ligation, followed by exonuclease treatment and PCR amplification all in a single tube. The entire procedure can be completed in two days with minimal hands-on time.

While the sample preparation steps are well-documented, processing the data is non-trivial. Once sequenced and aligned, the synthesized arms must be removed so that synthesis errors are not mistaken for genetic variation in a sample.

\section{APPROACH}

We developed a simple-to-use application that uses BWA mem ( $\mathrm{Li}, 2013)$ to map the sequence reads, then strips the ligation and extension arms and marks duplicate the reads using a unique molecular identifier (UMI). The application is written in python; it outputs alignments in BAM format (Li et al., 2009) and generates a report that includes the target enrichment and percent of on-target reads.

*To whom correspondence should be addressed. Email:
bpederse@gmail.com

\section{METHODS}

bwa-mips depends on bwa mem (Li, 2013) and Picard tools (http:// picard. sourceforge. net/). It accepts paired-end fastq files with an optional unique molecular identifier (UMI) at the start of the second read of each pair. Before streaming the sequences to bwa mem, bwa-mips moves the UMI into the read name so that it can be used later to mark duplicate reads. Once bwa mem has aligned the reads, bwa-mips removes the ligation and extension arms from the reads guided by the MIP's design file which contains the columns: ext_probe_start, ext_probe_stop, lig_probe_start and lig_probe_stop and chr. This matches the output of MIPgen, a recent piece of software to design MIPs (Boyle et al., 2014). The strand and order (first or second) of the read are used to lookup the MIP in the design file. Reads not found to be associated with a MIP are marked with a flag indicating that they failed QC, but they are still reported. Reads associated with a MIP have their sequence, quality, and cigar fields adjusted to remove arms so that synthesis errors are not present in the final aligned sequence. The steps are performed in this order because the read must be mapped before it is possible to know which MIP it belongs to.

The software accounts for insertions and deletions when adjusting both the sequence and the cigar string during arm removal and it adds several tags (Li et al., 2009) indicating the MIP index and the original sequence. Finally, if UMI's were added during the library preparation, the software marks duplicate reads. Of pairs with the same positions and UMIs, all but the one with the highest mapping quality are marked as duplicates.

Once the alignment is completed, a report indicating the percent of ontarget reads and the fold-enrichment (relative to what is expected by chance given the size of the target regions relative to the size of the genome) is shown.

The pipeline is enumerated in Table 1

\subsection{Example Usage}

Included in the bwa-mips repository is a full example. It includes a MIP design file for a region of chromosome 6 that is compatible with the output from MIPgen (Boyle et al., 2014). Once the reference genome is indexed with $b w a$, the entire pipeline can be run as

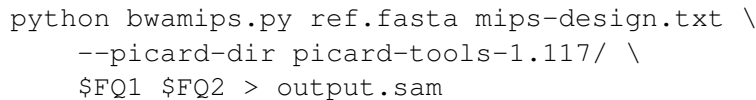

Where $\$ F Q 1$ and $\$ F Q 2$ are the paired end FASTQ files. The output.bam file will contain the alignments in BAM format. For this selected dataset, contained in the example/ directory of the bwa-mips repository, the output report indicates a 3000-fold enrichment of reads to the target region with $86 \%$ of reads mapped on-target. 
bioRxiv preprint doi: https://doi.org/10.1101/007260; this version posted July 19, 2014. The copyright holder for this preprint (which was not certified by peer review) is the author/funder, who has granted bioRxiv a license to display the preprint in perpetuity. It is made available under aCC-BY-ND 4.0 International license.

\section{Pedersen et al}

Table 1. steps in bwa-mips

\begin{tabular}{ll}
\hline step & explanation \\
\hline move UMI & $\begin{array}{l}\text { UMI is moved from the start of the 2nd read } \\
\text { of each pair to the read-name and reads are } \\
\text { streamed to bwa mem } \\
\text { bwa mem is used to align reads } \\
\text { the location and strand of the reads are used to } \\
\text { alignment }\end{array}$ \\
lookup the MIP from the design file \\
positions in the design file inform trimming of \\
the alignments such that arms (with potential \\
synthesis errors) are not included \\
the cigar string is adjusted to mirror the \\
trimming and SAM tags are added indicating \\
the MIP that each alignment belongs to. \\
alignments with the same start and UMI are \\
marked as duplicates \\
summary report of alignment statistics is \\
generated
\end{tabular}

\section{DISCUSSION}

While traditional enrichment procedures are well established, the requirement of mechanical fragmentation and size selection create bottlenecks as sequencing projects are scaled to thousands of samples (Shendure and Ji, 2008). Additionally, due to the upfront library construction, the cost structure of these procedures does not change much whether capturing $100 \mathrm{~kb}$ or $6 \mathrm{Mb}$. Together these show the importance of software to align MIP data.

While there has been much work to date on designing (Stenberg et al., 2005; Boyle et al., 2014) and using molecular inversion probes (Hardenbol et al., 2003, 2005), there are few tools that will map the resulting data. We have introduced bwa-mips to fill this void.

\section{CONCLUSION}

We have introduced bwa-mips, described its implementation and demonstrated its usage on an example dataset.

\section{ACKNOWLEDGEMENT}

Thanks to Evan Boyle and Jay Hesselberth for helpful discussions.

Funding: Text Text Text Text Text Text Text Text.

\section{REFERENCES}

Boyle, E. A., O'Roak, B. J., Martin, B. K., Kumar, A., and Shendure, J. (2014). Mipgen: Optimized modeling and design of molecular inversion probes for targeted resequencing. Bioinformatics, page btu353.

Hardenbol, P., Banér, J., Jain, M., Nilsson, M., Namsaraev, E. A., Karlin-Neumann, G. A., Fakhrai-Rad, H., Ronaghi, M., Willis, T. D., Landegren, U., et al. (2003). Multiplexed genotyping with sequence-tagged molecular inversion probes. Nature biotechnology, 21(6), 673-678.

Hardenbol, P., Yu, F., Belmont, J., MacKenzie, J., Bruckner, C., Brundage, T., Boudreau, A., Chow, S., Eberle, J., Erbilgin, A., et al. (2005). Highly multiplexed molecular inversion probe genotyping: over 10,000 targeted snps genotyped in a single tube assay. Genome research, 15(2), 269-275.

Li, H. (2013). Aligning sequence reads, clone sequences and assembly contigs with bwa-mem. arXiv preprint arXiv:1303.3997.

Li, H., Handsaker, B., Wysoker, A., Fennell, T., Ruan, J., Homer, N., Marth, G., Abecasis, G., Durbin, R., et al. (2009). The sequence alignment/map format and samtools. Bioinformatics, 25(16), 2078-2079.

Shendure, J. and Ji, H. (2008). Next-generation dna sequencing. Nature biotechnology, 26(10), 1135-1145.

Stenberg, J., Nilsson, M., and Landegren, U. (2005). Probemaker: an extensible framework for design of sets of oligonucleotide probes. BMC bioinformatics, $\mathbf{6}(1)$, 229 